Editorial

\title{
II Jornadas Nacionales de Formación y Desarrollo Profesional de Enfermería del Trabajo
}

\author{
II Occupational Health Nursing National Conference: Training and Professional \\ Development
}

\author{
Esperanza M. Alonso Jiménez \\ Consejería de Sanidad del Principado de Asturias. España. \\ Recibido: 03-11-14 \\ Aceptado: 18-11-14 \\ Correspondencia: \\ Esperanza M. ${ }^{a}$ Alonso Jiménez \\ Epidemiología Laboral y Ambiental \\ Dirección General de Salud Pública \\ Consejería de Sanidad del Principado de Asturias \\ C/ Ciriaco Miguel Vigil \\ 33006 Oviedo. España. \\ Teléfono 985.10.63.23. Ext 17476 \\ Correo electrónico: esperanzamaria.alonsojimenez@asturias.org
}

La Federación Española de Enfermería del Trabajo (FEDEET) se constituyó hace unos 5 años, con la idea inicial de unir a las distintas Asociaciones y Sociedades Científicas de Enfermería del Trabajo y Salud Laboral que había en el territorio nacional. Su objetivo principal ha sido y es la integración de la especialidad de Enfermería del Trabajo en los distintos ámbitos en los que desarrollan estos profesionales sus competencias y su actividad, tanto en Prevención de Riesgos Laborales, como en la Vigilancia de la Salud.

Desde el principio de nuestra constitución hemos venido celebrando y organizando distintos eventos en el territorio nacional de distinta envergadura, Jornadas, Congreso Nacional e Internacional, Seminarios en Aragón, Asturias, Cataluña, Cantabria y Madrid, entre otros, a través y en cooperación con las distintas Asociaciones y Sociedades que constituyen FEDEET. Este año se ha celebrado la II JORNADA NACIONAL DE FORMACIÓN Y DESARROLLO PROFESIONAL DE ENFERMERÍA DEL TRABAJO en Andalucía.

Esta II JORNADA "FEDEET" se ha centrado en una de las cinco grandes áreas de competencias profesionales específicas de los Enfermeros Especialistas en Enfermería del Trabajo: la de «Docencia e Investigación", actividades que resultan indispensables para el crecimiento y el desarrollo profesional de todos los Enfermeros del Trabajo, ya que tanto la actividad docente como la investigadora de la Enfermería del Trabajo redundarán en el logro del estatus de "disciplina autónoma" que perseguimos y que difícilmente se alcanzaría sin el desarrollo de estas competencias.

La Jornada se clausuró con una Ponencia que se hizo eco de las ideas principales desarrolladas a lo largo del día:

- La necesidad de desarrollar plenamente TODAS nuestras competencias profesionales, fijadas en el propio programa de nuestra especialidad y agrupadas en cinco grandes áreas que me van a permitir recordarles: Preventiva, Asistencial, Legal y pericial, de Gestión y Docente e Investigadora.

- Recalcar que los enfermeros especialistas del trabajo, sin perjuicio de los principios de interdisciplinariedad y multidisciplinariedad aplicables a los equipos profesionales que actúan en el ámbito de la salud laboral, ejercerán su profesión con plena AUTONOMÍA TÉCNICA Y CIENTÍFICA. 
- Que, para ello deben adquirir y mantener las competencias necesarias para el adecuado ejercicio de sus funciones en los distintos ámbitos de actuación de la especialidad, como recoge textualmente la Orden SAS/1348/2009, de 6 de mayo, por la que se aprueba y publica el programa formativo de la especialidad de Enfermería del Trabajo.

- Que las nuevas competencias de la enfermería del trabajo escritas, en el programa de la especialidad no le quitan nada a nadie, no inventan nuevas funciones..., sino que lo que se hace es ejercer nuestro derecho de desarrollar nuestras competencias con plena autonomía.

- Y por eso es necesario y casi obligatorio el deber de aprovechar esta oportunidad, asumiendo esa responsabilidad y autonomía en todos nuestros ámbitos de competencia, sin dejar fisuras y ocupando totalmente cada una de las cinco áreas de competencia.

En definitiva, que tenemos muchas cosas que hacer y que nos corresponde a nosotros hacerlas, hacerlas con convicción para que la Enfermería del Trabajo se sitúe en el lugar que le pertenece y por los propios enfermeros que es a quien le compete contarla.

En este sentido se recordó que la autonomía no se adquiere de forma gratuita sino que implica previamente un proceso de maduración, formación, crecimiento individual y como colectivo, para poder ejercitarse de forma plena. Y en esta línea de acción, sin duda alguna, la estrella de la Jornada fue el Área Docente e Investigadora, que no sólo es un área más de competencias, sino que se erige en una de las estrategias clave para el desarrollo de la profesión.

La investigación enfermera que ya se aborda desde la etapa formativa de grado, está dotando a los futuros profesionales de los conocimientos y de las herramientas básicas necesarias para que ésta sea una práctica habitual reconocida e integrada como el resto de conocimientos. Enfermero del Trabajo, por el papel clave que desempeña, requiere ocupar un lugar relevante en todos los niveles de organización y de la toma de decisiones. La investigación ofrece nuevas oportunidades para el crecimiento profesional y desarrollo competencial distintos de los meramente asistenciales. Tener competencias en el Área preventiva, promoción de la salud en la empresa, propician el liderazgo de la enfermera en los procesos de salud, repercutiendo en un mayor reconocimiento social; por eso se potencia con la figura del Enfermero del Trabajo "Investigador» que hará de referente del trabajador y de la empresa. A lo largo de las jornadas se ha consolidado el perfil del enfermero del trabajo cada vez más autónomo y exigente, con más acceso a la información y más capacidad para tomar decisiones y asumir responsabilidades.

Se consolidó la idea de que el Enfermero del trabajo debe realizar muchas y diversas actividades: asesorar a la empresa en el «cuidado» de los trabajadores, fomentar la cultura preventiva, interactuar con los representantes de los trabajadores, proponer medidas preventivas una vez que los riesgos sean evaluados, participar activamente en la selección de EPIs, realizar el seguimiento y control de los residuos, etc., porque su competencia en el área de Gestión le permite plantearse el liderazgo, por ello en la Jornada se habló de Coaching como herramienta de fortaleza y ayuda avanzar tanto personal como profesionalmente, de la actitud colaborativa con el resto de profesionales que conforman el Servicio de Prevención y no de aislamiento, interrelacionar y cooperar con el resto de profesionales que forman el equipo multidisciplinar sirviendo de vínculo para la mejora de muchas parcelas profesionales y también de la investigación multiprofesional. La transformación profesional que se vino comentada a lo largo de toda la Jornada no olvido el Área Legal y Pericial que, por sí misma, supone una enorme conquista y una puesta en valor de la profesión, sino que abre un campo de trabajo extensísimo y novedoso que aborda cuestiones basadas en evidencia científica, documentada en hechos investigados a través de método científico y que permiten al profesional de enfermería del trabajo elaborar informes de cambio de puesto de trabajo, de asesoramiento y orientados a la gestión de incapacidad, de asesoramiento y de reconocimiento del origen laboral de patologías, así como el peritaje y valoración de cuidados en sí mismo. Para ello es importante la incidencia en la construcción y la realización de estudios, protocolos y guías de buenas prácticas, gestionar y organizar los exámenes de salud, custodiar los registros sanitarios, gestionar redes y recursos informáticos de apoyo, garantizar la protección de datos, controlar la trazabilidad de medicamentos y productos, coordinación burocrática con el SNS, Mutuas y otros organismos.

Con las comunicaciones y ponencias presentadas durante la Jornada se debatieron estos y otros temas de interés y actualidad:

- El momento actual por el que está pasando la Enfermería es importantísimo una vez reconocida la formación en Enfermería del Trabajo como una especialidad en Ciencias de la Salud, encontramos 
frente a la realidad de la incorporación de enfermeros residentes a las Unidades Docentes Multiprofesionales de Salud Laboral que nacen al amparo de lo dispuesto en el Real Decreto 183/2008 y que formarán tanto a médicos como enfermeros del trabajo, consolidando la colaboración multidisciplinar de forma simultánea a la asunción progresiva de la responsabilidad inherente al ejercicio autónomo de la profesión enfermera.

- Los nuevos especialistas son los protagonistas del desarrollo de la profesión que, día a día, tienen que hacer valer la excelente preparación para consolidar todas las áreas de competencia que el enfermero del trabajo desarrolla en su actividad y que esto casi nunca es fácil, ya que a veces, parece que seamos nosotros mismos los que no vemos lo que está escrito en nuestro programa, o que no queramos verlo, esperando a que nos digan qué hacer, cómo hacerlo y cuándo. Que sean ellos (los noveles especialistas) quienes se den a valer.

- No se puede esperar que esa "plena autonomía técnica y científica", esa "responsabilidad en la toma de decisiones", la capacidad de decidir sobre lo que pueden hacer los Enfermeros Especialista en Enfermería del Trabajo, venga "llovido del cielo", tampoco por el mero hecho de poseer vastos conocimientos o disponer de mucha información respecto a algo, o se nos reconozcan determinadas competencias, vayamos a obtener todos los beneficios que podían esperarse de ese conocimiento o reconocimiento.

Ha sido gratificante ver y formar parte de esta experiencia en la que la enorme potencialidad que la Enfermería del Trabajo tiene ha quedado reflejada en los trabajos y comunicaciones presentados tanto por ponentes como por asistentes; pero es necesario recordar que como todas las potencialidades, de nada sirven si no se materializan, que todo lo que podamos y estemos capacitados para hacer, pero no hagamos, alguien lo hará, y lo hará en lugar de nosotros y sin contar con nosotros.

Que debemos abandonar actitudes indolentes; debemos tener la clara voluntad de hacer las cosas. Y para ello, contamos con una ayuda muy importante: en el Programa de nuestra especialidad se nos reconoce la capacidad de hacerlas. "No tenemos que ganarnos nada. Lo que debemos hacer es evitar perderlo,".

Hemos dado un salto cualitativo en la especialidad; mantenemos la esencia del "Enfermero de Empresa", tenemos más personalidad profesional y un programa formativo sólido, basado en conocimientos científicos, con nuevas competencias que nos permiten dar un salto cualitativo, estas nuevas competencias reflejadas con la inclusión de las áreas Legal y Pericial, junto con la de Gestión, hacen que no exista ningún campo de actuación en el que pueda ponerse en duda la AUTONOMÍA del Enfermero Especialista en Enfermería del Trabajo.

Hace falta que la llama siga viva y que la especialidad se consolide. Para ello y a modo de conclusiones sirvan las siguientes reflexiones que surgieron en las Jornadas:

- El importantísimo papel que la Enfermería del Trabajo tiene encomendado en esos equipos interdisciplinares que constituyen los Servicios de Prevención.

- Debemos ser conscientes que no es fácil superar, demostrar y salvar dificultades como estamos fuera del sistema nacional de salud y nuestra área es desconocida para la inmensa mayoría de otras especialidades.

- Estamos generando el concepto de colectivo y eso es bueno para el desarrollo y transformación profesional de la enfermería del Trabajo.

- Y que las herramientas como la investigación, la docencia, la gestión, etc., las tenemos que desarrollar por completo.

En definitiva, ya hemos cambiado desde el punto de vista formativo, ahora vamos a cambiar desde el punto de vista de imagen que generamos a través del alcance de nuestra actividad y en particular a través del colectivo profesional de enfermeros/as del trabajo (ya que las competencias las tenemos escritas en el cuerpo disciplinar como propias de la profesión de enfermería del trabajo) y solo nosotros podemos hacer ese cambio de imagen.

Para terminar queremos recordar que la FEDEET tiene abiertas las puertas a todos aquellos profesionales de enfermería que trabajen en Salud Laboral, Vigilancia de la salud y/o prevención de Riesgos laborales, que desde la FEDEET se defiende a sus asociados y que creemos necesario asociaciones y federaciones fuertes en materia de profesionales de la Vigilancia de la Salud, por la que invitamos a todos los enfermeros del trabajo a formar parte de proyecto de FEDEET y a no quedaros aislados y al margen. 
Quisiera aprovechar este último párrafo para agradecer a todos y cada unos de los enfermeros del trabajo que estuvieron en Sevilla el esfuerzo de asistir, de estar y de ser, así como a todos aquellos socios que se quedaron con las ganas de ir y no pudieron por diversas razones. A los ponentes invitados por regalarnos su tiempo y compartir conocimiento con todos los que estábamos allí. Al Colegio de Enfermería de Sevilla que tan generosamente nos cedió sus instalaciones y su personal durante la Jornada, y en especial a la Junta Directiva de FEDEET que hace que esto sea posible con su apoyo incondicional, el mismo que nos presta la Escuela Nacional de Medicina del Trabajo del Instituto de Salud Carlos III, cada vez que compartimos con ellos un proyecto o una Jornada como esta.

Y un agradecimiento especial al Sr. Ángel María Martín que me ha dejado usar partes de su conferencia de clausura para este editorial. 